\title{
Adverse Health Consequences of Dietary Advanced Glycation End Products (ages) and Inhibitory Effects of Natural Ingredients on Ages
}

\author{
Zihao Wei and Qingrong Huang* \\ Department of Food Science, Rutgers University, USA
}

Received: October 11,2017; Published: October 16, 2017

*Corresponding author: Qingrong Huang, Department of Food Science, Rutgers University, 65 Dudley Road, New Brunswick, New Jersey 08901, United States, Tel: +1 (848) 932-5514; Fax: +1 (732) 932-6776; Email: qhuang@sebs.rutgers.edu

\begin{abstract}
Dietary advanced glycation end products (AGEs) may result in various adverse health consequences, such as inflammation, cancer, liver diseases, obesity, cardiovascular diseases, diabetes, proteostatis impairment and memory decline. Natural ingredients may be a promising strategy to inhibit AGEs generation and ameliorate health risks of AGEs. The mechanisms involved in inhibition of AGEs with use of natural ingredients include antioxidant activity, reactive carbonyl trapping, and suppressing pathways of RAGE (receptor of advanced glycation end product), MAPK (mitogen-activated protein kinases) and NF- $\mathrm{kB}$ (nuclear factor-kappa B).This mini review summarizes the present understanding of the adverse impact of AGEs on health and the inhibitory effect of natural ingredients on AGEs.
\end{abstract}

Keywords: Maillard reaction; Dietary advanced glycation end products; Adverse health consequences; Inhibitor; Natural ingredients

Abbreviations: AGEs: Advanced Glycation End Products; NAFLD: Non-Alcoholic Fatty Liver Disease; CML: Carboxy Methyl Lysine; RAGE: Receptor of Advanced Glycation End Product; MAPK: Mitogen Activated Protein kinases; LSOPC: Lotus Seedpod Oligomeric Pro Cyanidins

\section{Introduction}

Maillard reaction, a form of non-enzymatic browning, is a chemical reaction between proteins and reducing sugars in vitro and in vivo [1,2]. The Maillard reaction consists of three stages: the early, advanced and final stages. Briefly speaking, the initial glycosylation reaction occurs during the early stage of the Maillard reaction, and amadori products are formed through rearrangement of Schiff bases [3]. For the advanced stage, the Amadori products subsequently form cross-linked structures with a high degree of complexity named advanced glycation end products (AGEs) through fission, dehydration and Strecker degradation [1]. In terms of final stage, nitrogenous polymers and co-polymers termed melanoidins are formed [1]. Although Maillard reaction may aid in development of attractive color, flavor and aroma in food products, it is worth pointing out that AGEs in Maillard reaction products may lead to adverse health consequences [4,5]. In recent years extensive research reveals that AGEs are associated with various diseases such as cancer and diabetes $[4,6]$. Foods are major sources of AGEs, and commonly consumed foods such as grilled chicken, broiled steak and French fries contain relatively high content of AGEs [7]. Meanwhile, a recent study indicates that a high level of dietary AGEs is associated with a high level of AGEs in plasma and urine [8]. Because contents of AGEs derived from diet cannot be ignored and AGEs in the diet are associated with induction and progression of many chronic diseases, it is essential to comprehensively understand adverse health consequences of dietary AGEs.

In view of numerous health risks of dietary AGEs, multiple measures are required to suppress AGEs. Medicine such as metformin and irbesartan can be applied to relieve adverse health effects induced by AGEs [9]. However, the synthetic pharmaceutical ingredients may bring about short-term health risks. Metformin may lead to digestive disorders such as vomiting and diarrhoea and irbesartan can sometimes result in symptoms such as dizziness and headache $[10,11]$. Therefore, considering the side effects of synthetic ingredients, natural ingredients should be employed as AGEs inhibitors in place of synthetic ingredients. During recent decade's inhibition of AGEs with natural ingredients have drawn substantial scholarly attentions. More and more natural ingredients prove to possess inhibitory influence on AGEs, and significant achievements about the underlying molecular mechanisms have been made $[3,12,13]$. Thus, the present mini-review aims to address the current understandings towards adverse health consequences of dietary AGEs and inhibitory influence of natural ingredients on AGEs. 


\section{Adverse Health Consequences of Dietary Advanced Glycation End Products}

Extensive biomedical studies about health effects of dietary AGEs on animals and human beings have been conducted over the past few years $[14,15]$. As shown in Figure 1, relevant research indicates that dietary AGEs are closely linked to induction and progression of diverse diseases such as inflammation, cancer, liver diseases, obesity, cardiovascular diseases, diabetes, proteostatis impairment and memory decline. It is worthwhile to note that the adverse health consequences of dietary AGEs depend on actuation duration. At times short-term intake of dietary AGEs may not have profound health risks. A recent study revealed that shorttem ingestion of advanced AGEs could possibly exert little health influence on rats [16].

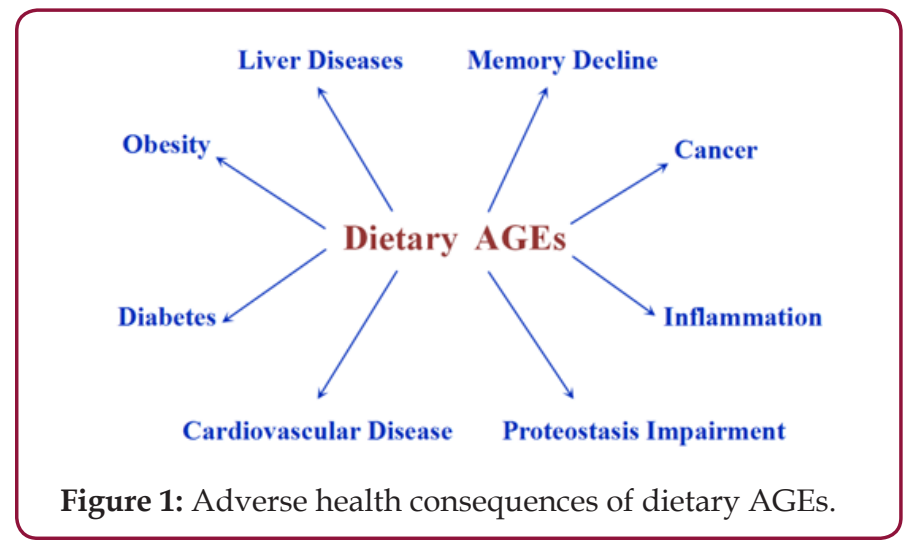

\section{Inflammation}

Several studies support the association between dietary AGEs and inflammation. Based on accurate analysis of AGEs content in the diet, a cross-sectional study divided participants into two groups: high and low intake of dietary AGEs. During the 3-month period a correlation between intake of dietary AGEs and inflammation was observed, indicating that a chronic AGEs-rich diet could result in inflammation [5]. In addition, it was reported that long-term consumption of AGEs in thermally processed red meat could lead to chronic inflammation [4].

\section{Cancer}

Recent studies reveal that dietary AGEs can increase risk of certain cancers. After analysis of more than 2000 human pancreatic cancer cases during an average of 10.5-year follow-up, it was found that higher intake of dietary AGEs contributed to higher risk of pancreatic cancer in men, and consumption of dietary AGEs was not linked to risk of pancreatic cancer in women [4].

\section{Liver diseases}

AGEs in food may induce various liver diseases including liver injury, liver fibrosis, liver inflammation and so on. In mice model of NAFLD (non-alcoholic fatty liver disease), long-term exposure to high levels of dietary AGEs worsened liver pathology through modulating liver injury and liver fibrosis, and reducing intake of dietary AGEs might weaken the harmful effects [14]. NE(carboxymethyl) lysine (CML) is one of the major dietary AGEs, and oral acute and sub acute toxicity of CML was evaluated in mice models. It was found that CML presented negligible acute hazards, but repeated ingestion of CML could induce liver damage via oxidative stress [15]. Influence of dietary AGEs on liver was studied using a mice model, and the results suggested that diets with high levels of AGEs could induce liver inflammation in the absence of steatosis [17].

\section{Obesity and cardiovascular diseases}

Obesity is closely associated with cardiovascular diseases, and they are both major health problems [18]. Recent studies have demonstrated that dietary AGEs can bring about obesity and cardiovascular diseases. Different groups of mice were fed with diets containing high or low levels of AGEs, and weight gain of these mice was analyzed after 6 weeks. It was observed that a highAGEs diet could contribute to weight gain [19]. Although evidence is still lacking about links between dietary AGEs in children and adolescents, sufficient data demonstrate that dietary AGEs can induce vascular dysfunction in adults, contributing to occurrence of cardiovascular diseases [20].

\section{Diabetes and proteostatis impairment}

A large amount of evidence suggests that dietary AGEs may interfere with metabolism of sugars and proteins, leading to diabetes and proteostatis impairment. Mice were treated with oral AGEs, and a series of indexes such as glucose tolerance, glucose uptake and gene modulation were analyzed. It was found that chronic ingestion of AGEs promoted insulin resistance and diabetes, and the relevant mechanism was possibly depleting the antioxidant defenses AGE receptor-1 and sirtuin-1 [6]. In vivo studies revealed that chronic intake of dietary AGEs may cause disruption of proteostasis through inhibiting proteasome peptidase activity [21].

\section{Memory decline}

Correlations exist between dietary AGEs and cognitive decline. High oral intake of AGEs was reported to be associated with faster rate of memory decline in young elderly. To avoid disruption of daily life by memory loss, the intervention method of taking a lowAGEs diet may reduce risk of cognitive compromise [22].

\section{Inhibiting formation of Advanced Glycationend Products with Natural Ingredients}

Given that AGEs can bring about potential health risks, various measures should be taken to suppress formation of AGEs. During recent years natural ingredients prove to be a reliable means to reduce contents of AGEs. Due to high biosafety and biocompatibility of natural ingredients, inhibiting AGEs with natural ingredients has attracted scientific and industrial interests in many fields. In general, natural ingredients can suppress AGEs formation via mechanisms such as antioxidant activity, reactive carbonyl trapping, and disruption of RAGE-MAPK signaling and NF- $\mathrm{BB}$ activation.

\section{Antioxidant Activity}

It is reported that many natural ingredients have powerful antioxidant activity, which helps to scavenge free radicals. Multiple studies demonstrate that inhibitory influence of some natural ingredients is ascribed to their antioxidant activity. Influence of 
ferulic acid on in vitro glycation of soy proteins was investigated utilizing $\mathrm{N} \varepsilon$-(carboxymethyl) lysine (CML) analysis and fluorescence measurement. It was found that use of ferulic acid could reduce formation of CML and fluorescent AGEs by around 90\% [3]. Recent work investigated inhibitory effects of ethanolic extracts from 17 medicinal plants on glycation of bovine serum albumin, and total phenolic content and antioxidant capacity of the medical plant extracts were also studied. The results demonstrated that influence of medical plant extracts on AGEs inhibition was positively correlated with free radical scavenging activity, indicating that the antioxidant activity of medical plant extracts contributed largely to their antiglycation effects [23].

A 4-week study revealed that red grape skin extract at different concentrations (0.031-0.500 mg/mL) could suppress formation of AGEsduringrelativelylong-termexposuretoAGEs [24].Incomparison with red grape skin extract, MesonaChinensisBenth extract required higher effective concentrations. MesonaChinensisBenth extract at different concentrations (0.25-1.00 mg/mL) significantly inhibited AGEs formation during 4 weeks of study [25]. In addition, olive leaf extract could inhibit formation of AGEs in a biscuit model, and the main way of action was possibly inhibiting oxidation of reaction intermediates towards the AGEs formation [26]. Apart from these in vitro studies, in vivo studies also indicate that antioxidant activity of certain natural ingredients is associated with their inhibitory effects on AGEs. Influence of Cuminumcyminumon AGEs inhibition in diabetic rats was systematically investigated, and the results showed that Cuminumcyminum could control oxidative stress and suppress formation of AGEs. The in vivo inhibitory effects of Cuminumcyminumon AGEs proved to be correlated with free radicals scavenging of Cuminumcyminum [27].

\section{Reactive Carbonyl Trapping}

Among current published literature reactive carbonyl trapping is the major mechanism involved in inhibition of natural ingredients on AGEs. Methylglyoxal and glyoxal are major carbonyl compounds during Maillard reaction and identified as well-known precursors of AGEs, and trapping these reactive carbonyl compounds may reduce formation of AGEs [28]. A recent study demonstrated that anthocyanins in blackcurrant could react with 50 percent of methylglyoxal when blackcurrant anthocyanins were incubated with methylglyoxal, suggesting that blackcurrant anthocyanins could effectively prevent AGEs formation by trapping methylglyoxal [29].

Polyphenols in Houttuyniacordata consisted of components quercitrin, chlorogenic acid and rutin. It was found that polyphenols in Houttuyniacordata could decrease AGEs production by 91$94.6 \%$ [28]. Genistein is a natural isoflavone derived from soy products, and genistein could inhibit AGEs production via trapping reactive dicarbonyl species efficiently under in vitro physiological

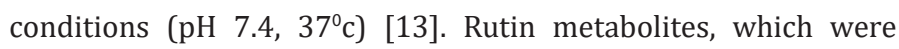
formed through metabolism of rutin by gut microflora, could serve as effective natural inhibitor of AGEs by tapping both methylglyoxal and glyoxal [30]. Since rutin is a commonly consumed flavonoid that is rich in many fruits and vegetables, the use of rutin metabolites may be a feasible way to attenuate AGEs formation. Pomegranate phenolics and their in vivo metabolites had inhibitory effects on all three stages of Maillard reaction (early, middle and late stages), and pomegranate phenolics could significantly suppress AGEs production via scavenging reactive carbonyl species such as methylglyoxal [31].

Since pomegranate is a fruit with good flavors and odors, oral intake of pomegranate appears an attractive dietary strategy to prevent and offset adverse health consequences of AGEs. Anti glycation effects of phenolic phyto chemicals extracted from blueberries, blackberries, strawberries, raspberries, cranberries and grapes were investigated, and the results showed that these sugar-free phyto chemicals could suppress AGEs generation by scavenging reactive carbonyls [32]. In addition, natural ingredients such as chlorogenic acid, ellagic acid, thiamin, quercetin, and oligomeric procyanidins of lotus seedpod, herbal teas and thymoquinone could effectively inhibit AGEs generation through reactive carbonyl trapping [12,33-38].

\section{Disruption of RAGE-MAPK Signaling and NF- $\kappa$ Bactivation}

Receptor of advanced glycation end product (RAGE), mitogenactivated protein kinases (MAPK) and nuclear factor-kappa B $(\mathrm{NF}-\mathrm{\kappa B})$ are detrimental pathways in animals and people, and a number of evidence reveals that inhibitory effects of natural ingredients on AGEs are associated with disruption of RAGEMAPK signaling and NF- $\mathrm{BB}$ activation. High-fat diet rats were selected as model to study inhibition of AGEs by lotus seedpod oligomericprocyanidins (LSOPC), and expression of RAGE, MAPK and NF- $\mathrm{KB}$ was investigated. It was found that LSOPC could inhibit formation of AGEs in rats through suppression of RAGE-MAPKNF- $\kappa B$ signaling [39]. Diabetic cardiomyopathy rats were applied to investigate influence of mangiferin on formation of AGEs, and the results demonstrated that mangiferin could effectively inhibit AGEs formation via NF- $\kappa \mathrm{B}$ deactivation and decreasing expression of RAGE [40].

\section{Concluding Remarks and Future Perspectives}

In summary, dietary AGEs may lead to the induction and progression of various health problems such as inflammation, cancer, liver diseases, obesity, cardiovascular diseases, diabetes, proteostatis impairment and memory decline. Natural ingredients may serve as an approach to reducing AGEs generation, and the involved inhibition mechanisms are antioxidant activity, reactive carbonyl trapping, and disruption of RAGE-MAPK signaling and $\mathrm{NF}-\kappa \mathrm{B}$ activation. Generally speaking, the natural ingredients are promising intervention tools to control content of AGEs. Meanwhile, we need to realize that there are many unexplored areas and ongoing challenges. Molecular mechanisms involved in health risks of dietary AGEs should be fully understood in details, and more in vivo animal and human studies should be conducted to confirm the inhibitory effects of natural ingredients on AGEs generation.

\section{References}

1. De Oliveira FC, Coimbra JSR, De Oliveira EB, Zuñiga ADA, Rojas EEG (2016) Food protein-polysaccharide conjugates obtained via the 
Maillard reaction: A review. Critical Reviews in Food Science and Nutrition 56(7): 1108-1125.

2. Tessier FJ (2010) The Maillard reaction in the human body. The main discoveries and factors that affect glycation. Pathologie Biologie 58(3): 214-219.

3. Silván JM, Assar SH, Srey C, Del Castillo, Ames JM (2011) Control of the Maillard reaction by ferulic acid. Food Chemistry 128(1): 208-213.

4. Jiao L, Stolzenberg-Solomon R, Zimmerman TP, Duan Z, Chen L, et al. (2015) Dietary consumption of advanced glycation end products and pancreatic cancer in the prospective NIH-AARP Diet and Health Study. The American Journal of Clinical Nutrition 101(1): 126-134.

5. Pino AD, Currenti W, Urbano F, Scicali R, Piro S, et al. (2017) High intake of dietary advanced glycationend-productsis associated with increased arterial stiffness and inflammation in subjects with type 2 diabetes. Nutrition, Metabolism \& Cardiovascular Diseases.

6. Cai W, Ramdas M, Zhu L, Chen X, Striker GE, et al. (2012) Oral advanced glycationendproducts (AGEs) promote insulin resistance and diabetes by depleting the antioxidant defenses AGE receptor- 1 and sirtuin 1 . Proceedings of the National Academy of Sciences 109(39): 1588815893.

7. Uribarri J, Woodruff S, Goodman S, Cai W, Chen X, et al. (2010) Advanced glycation end products in foods and a practical guide to their reduction in the diet. Journal of the American Dietetic Association 110(6): 911916.

8. Scheijen JLJM, Hanssen NMJ, Van Greevenbroek MM, Van der Kallen CJ, Feskens EJM, et al. (2017) Dietary intake of advanced glycation end products is associated with higher levels of advanced glycation end products in plasma and urine: The CODAM study. Clinical Nutrition.

9. Ishibashi Y, Matsui T, Takeuchi M, Yamagishi S (2012) Beneficial effects of metformin and irbesartan on advanced glycation end products (AGEs)RAGE-induced proximal tubular cell injury. Pharmacological Research 65(3): 297-302.

10. Bouchoucha M, Uzzan B, Cohen R (2011) Metformin and digestive disorders. Diabetes \& Metabolism 37(2): 90-96.

11. Croom KF, Plosker GL (2008) Irbesartan. Drugs 68(11): 1543-1569.

12. Li X, Zheng T, Sang S, Lv L (2014) Quercetin inhibits advanced glycation end product formation by trapping methylglyoxal and glyoxal. Journal of Agricultural and Food Chemistry 62(50): 12152-12158.

13. Lv L, Shao X, Chen H, Ho CT, Sang S (2011) Genistein inhibits advanced glycation end product formation by trapping methylglyoxal. Chemical Research in Toxicology 24(4): 579-586.

14. Leung C, Herath CB, Jia Z, Andrikopoulos S, Brown BE, et al. (2016) Dietary advanced glycation end-products aggravatenon-alcoholic fatty liver disease. World Journal of Gastroenterology 22(35): 8026-8040.

15. Liu X, Zheng L, Zhang R, Liu G, Xiao S, et al. (2016) Toxicological evaluation of advanced glycation end product $\mathrm{N} \varepsilon$-(carboxymethyl) lysine: Acute and subacute oral toxicity studies. Regulatory Toxicology and Pharmacology 77:65-74.

16. Poulsen MW, Andersen JM, Hedegaard RV, Madsen AN, Krath BN et al. (2016) Short-term effects of dietary advanced glycation end products in rats. British Journal of Nutrition 115(4): 629-636.

17. Patel R, Baker SS, Liu W, Desai S, Alkhouri R, et al. (2012) Effect of dietary advanced glycationend products on mouse liver. Plos One 7(4): e31543.

18. Marinou K, Tousoulis D, Antonopoulos AS, Stefanadi E, Stefanadis C (2010) Obesity and cardiovascular disease: From pathophysiology to risk stratification. International Journal of Cardiology 138(1): 3-8.

19. Sayej WN, Knight PR, Guo WA, Mullan B, Ohtake PJ, et al. (2016) Advanced glycation end products induce obesity and hepatosteatosis in CD-1 wildtype mice. Bio Med Research International 2016: 1-12.
20. Gupta A, Uribarri J (2016) Dietary advanced glycationend products and their potential role in cardio metabolic disease in children. Hormone Research in Paediatrics 85(5): 291-300.

21. Tsakiri EN, Iliaki KK, Höhn A, Grimm S, Papassideri IS, et al. (2013) Diet-derived advanced glycation end products or lipofuscin disruptsproteostasis and reduces life span in Drosophila melanogaster. Free Radical Biology and Medicine 65: 1155-1163.

22. West RK, Moshier E, Lubitz I, Schmeidler J, Godbold J, et al. (2014) Dietary advanced glycation end products are associated with declinein memory in young elderly. Mechanisms of Ageing and Development 140: 10-12.

23. Harris CS, Beaulieu LP, Fraser MH, McIntyre KL, Owen PL, et al. (2011) Inhibition of advanced glycation end product formation by medicinal plant extracts correlates with phenolic metabolites and antioxidant activity. Planta Medica 77(2): 196-204.

24. Jariyapamornkoon N, Yibchok-anun S, Adisakwattana S (2013) Inhibition of advanced glycation end products by red grape skin extract and its antioxidant activity. BMC Complementary and Alternative Medicine 13: 171.

25. Adisakwattana S, Thilavech T, Chusak C (2014) MesonachinensisBenth extract prevents AGE formation and protein oxidation againstfructoseinduced protein glycation in vitro. BMC Complementary and Alternative Medicine 14: 130 .

26. Navarro M, Morales FJ (2017) Effect of hydroxytyrosol and olive leaf extract on 1,2-dicarbonylcompounds, hydroxyl methyl furfural and advanced glycation end products in a biscuit model. Food Chemistry 217: 602-609.

27. Jagtap AG, Patil PB (2010) Antihyperglycemic activity and inhibition of advanced glycation end product formation by Cuminumcyminum in streptozotocin induced diabetic rats. Food and Chemical Toxicology 48(8-9): 2030-2036.

28. Yoon SR, Shim SM (2015) Inhibitory effect of polyphenols in Houttuyniacordata on advancedglycationend-products (AGEs) by trapping methylglyoxal. LWT-Food Science and Technology 61(1): 158163.

29. Chen XY, Huang IM, Hwang LS, Ho CT, Li S, et al. (2014) Anthocyanins in blackcurrant effectively prevent the formation of advanced glycation end products by trapping methylglyoxal. Journal of Functional Foods 8: 259-268.

30. Pashikanti S, De Alba, Boissonneault GA, Cervantes-Laurean D (2010) Rutin metabolites: Novel inhibitors of nonoxidative advanced glycation end products. Free Radical Biology \& Medicine 48(5): 656-663.

31. Liu W, Ma H, Frost L, Yuan T, Dain JA (2014) Pomegranate phenolics inhibit formation of advanced glycationendproducts by scavenging reactive carbonyl species. Food \& Funcion 5(11): 2996-3004.

32. Wang W, Yagiz Y, Buran TJ, Nunes CN, Gu L (2011) Phytochemicals from berries and grapes inhibited the formation of advancedglycation endproducts by scavenging reactive carbonyls. Food Research International 44(9): 2666-2673.

33. Hori M, Yagi M, Nomoto K, Shimode A, Ogura M, et al. (2012) Inhibition of advanced glycation end product formation by herbal teas and its relation to anti-skin aging. Anti-Aging Medicine 9(6): 135-148.

34. Kim J, Jeong I, Kim CS, Lee YM, Kim JM, et al. (2011) Chlorogenicacid inhibits the formation of advanced glycationend products and associated protein cross-linking. Archives of Pharmacal Research 34(3): 495-500.

35. Losso JN, Bawadi HA, Chintalapati M (2011) Inhibition of the formation of advanced glycation end products by thymoquinone. Food Chemistry 128(1): 55-61.

36. Muthenna P, Akileshwari C, Reddy GB (2012) Ellagic acid, a new antiglycating agent: its inhibition ofN ${ }^{\varepsilon}$-(carboxymethyl)lysine. Biochemical Journal 442(1): 221-230. 
37. Srey C, Hull GLJ, Connolly L, Elliott CT, Del Castillo MD, et al. (2010) Effect of inhibitor compounds on $\mathrm{N}^{\varepsilon}$-(carboxymethyl) lysine(CML) and $\mathrm{N}^{\varepsilon}$-(carboxymethyl)lysine (CEL) formation in model foods. Journal of Agricultural and Food Chemistry 58(22): 12036-12041.

38. Wu Q, Chen H, Lv Z, Li S, Hu B, et al. (2013) Oligomericprocyanidins of lotus seedpod inhibits the formation of advanced glycationend-products by scavenging reactive carbonyls. Food Chemistry 138(2-3): 1493-1502.

39. Wu Q, Li S, Li X, Sui Y, Yang Y, et al. (2015) Inhibition of advanced glycationend product formation by lotusseedpod oligomericprocyanidins through RAGE-MAPK signaling and NF-kBactivation in high-fat-diet rats. Journal of Agricultural and Food Chemistry 63(31): 6989-6998.

40. Hou J, Zheng D, Fung G, Deng H, Chen L, et al. (2016) Mangiferin suppressed advanced glycation end products (AGEs) through NF$\kappa B d e a c t i v a t i o n$ and displayed anti-inflammatory effect in streptozotocin and highfat diet-diabetic cardiomyopathy rats. Canadian Journal of Physiology and Pharmacology 94(3): 332-340.

$\begin{array}{ll}\text { BIOMEDICAL } & \text { Assets of Publishing with us } \\ \text { RESEARCHES } & \text { Global archiving of articles } \\ & \text { - Immediate, unrestricted online access } \\ & \text { - Rigorous Peer Review Process } \\ & \text { - Authors Retain Copyrights }\end{array}$

